\title{
An Efficient Distance Estimation Algorithm for Indoor Sensor Network
}

\author{
P. T. V. Bhuvaneswari and V. Vaidehi
}

\begin{abstract}
Localization in sensor networks deals with the estimation of the position of the sensor node in a network for a given incomplete and inaccurate pair-wise distance measurements. Such distance data may be acquired by a sensor node by communicating with neighboring nodes called anchor nodes whose positions are known apriori. This paper proposes a Kalman filtering based distance estimation algorithm for indoor wireless sensor networks. In this paper, the distance of the unknown node is computed based on the Received Signal Strength (RSS) measurements. The effect of path loss and attenuation in the wireless medium are also considered in this proposed algorithm. The distance error is minimized using one-dimensional Kalman filter. The number of iterations in Kalman filter is limited using Cramer Rao Bound (CRB) value. A real-time experimentation is carried out to get Received Signal Strength value in indoor environment using zigbee series 1 RF module along with the associated X-CTU software of Maxstream. The proposed algorithm is simulated in MATLAB version 7. From the simulation results it is found that the proposed distance estimation algorithm gives accurate results.
\end{abstract}

Index Terms-Received signal strength, log normal shadowing model, ITU model, one-dimension Kalman estimator, cramer rao bound.

\section{INTRODUCTION}

Wireless sensor networks are fundamentally intended to provide information about the spatio-temporal characteristics of the observed physical world. Hence, it is necessary to associate sensed data with locations, making data geographically meaningful [1]. Hence, localization, a mechanism for autonomously discovering and establishing spatial relationships among sensor nodes, is of great importance in the development of wireless sensor networks. In most applications, the data reported by the sensors is relevant only if tagged with the accurate location of the sensor nodes. Thus knowledge of position of the node becomes inevitable.

The determination of location information requires the estimation of distance between the beacon nodes and unknown nodes. The beacon node is a node that is aware of its location while unknown node is the one whose locations is to be determined. The distance measurement between the beacon nodes and unknown nodes can be done by three techniques like Received Signal Strength (RSS), Time of Arrival (ToA) and Time Difference of Arrival (TDoA) [2],

Manuscript received October 9, 2011; revised December 15, 2011.

Authors are with Madras Institute of Technology, Anna University, Chennai, Tamilnadu, India (e-mail: ptvbmit@annauniv.edu, vaidehi@annauniv.edu)
[3]. However, distributed RSS-based distance measurement technique is more suitable considering the power constraints in the design of wireless sensor network.

In practice, due to resource constraints on the sensor nodes, additional hardware to perform distance measurements becomes infeasible. The commercially available zigbee RF modules provide the Received Signal Strength information through RSS Indicator (RSSI). In this paper, an efficient distance estimation algorithm based on RSS measurement is developed, which provides an accurate and cost-effective solution. Estimation of accurate distance is done by one-dimension Kalman filter estimator [4]. The number of iteration of the Kalman filter estimator is limited by Cramer-Rao Bound (CRB) [5].

The rest of the paper is organized as follows. Section II provides an overview of existing approaches. Section III presents the proposed algorithm with relevant mathematical formulations. Section IV presents the simulation results and performance analysis of the proposed algorithm. Finally, section $\mathrm{V}$ concludes the paper with future work.

\section{RELATED WORK}

The estimator determines the unknown node's locations with reference to the anchor node's positions. Reichenbach and Timmermann [6] proposes Received Signal Strength based localization algorithm with weighted centroid method for indoor wireless sensor network which offers low communication overhead and low computational complexity. But the reduction in RSS measurement errors is achieved by antenna diversity technique. This requires two antennas thus results in increase of hardware complexity. Prasan Kumar sahoo et al. [7] suggested a method of node localization from distance and angle measurements which requires more number of anchor nodes for achieving accuracy.

Zhao et al., [8] proposes a hop-distance algorithm for self-localization in WSN which is based on RSS and uses maximum likelihood estimator to achieve accuracy. Masashi Sugano et al., [9] propose a localization system based on zigbee standard which estimates the distance between sensor nodes by Received Signal Strength measurements. The computational cost is high due to maximum likelihood estimator and MMSE estimator. Shi Qin-Qin et al., [10] proposes a localization algorithm based on linear intersection which reduces the computational complexity but does not provide better accuracy. Thus, existing distance estimation methods provide better accuracy but with high computational cost. However the proposed algorithm aims to achieve good level of accuracy with reduced computational cost by employing one-dimensional Kalman filter estimator to estimate the distance. 


\section{Proposed Distance Estimation Algorithm}

Consider a sensor network comprising of $S_{n}$ nodes located at the coordinates $\left(x_{n}, y_{n}\right)$, where $\mathrm{n}$ represents the total number of nodes in the network. Assume that there are $S_{k}$ anchor nodes whose positions are known (eg. obtained via GPS or some other "absolute" reference) and that the remaining $\mathrm{S}_{\mathrm{u}}$ nodes are located at unknown positions. Without loss of generality, assume that position of nodes $S_{k+1} \ldots S_{n}$ are unknown, whereas the locations of the nodes $S_{1} \ldots S_{k}$ are known. Let $\theta_{1}, \theta_{2}$ be the coordinates of the nodes whose positions are known and unknown respectively.

$$
\begin{aligned}
& \theta_{I}=\left\{\left(\mathrm{x}_{\mathrm{k}}, \mathrm{y}_{\mathrm{k}}\right)\right\}, k=S_{I}, S_{2}, \ldots, S_{k} \\
& \theta_{2}=\left\{\left(\mathrm{x}_{\mathrm{u}}, \mathrm{y}_{\mathrm{u}}\right)\right\}, u=S_{k+l}, \ldots ., S_{n}
\end{aligned}
$$

The coordinates $\theta_{2}$ can be estimated with the knowledge of $\theta_{l}$ and samples of pair-wise range measurement $\left\{\gamma_{k u}{ }^{(t)}\right\}$ taken over $N$ different instances. In this paper, the pair-wise range measurement is computed through RSS-based range measurement method. The proposed distance estimation algorithm consists of four stages as shown in Fig. 1.

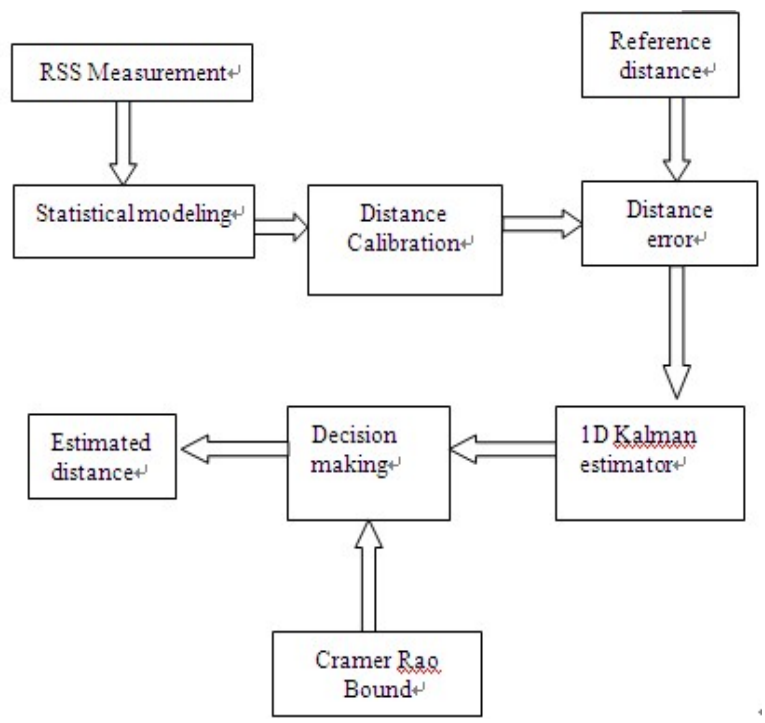

Fig. 1. Block diagram of distance estimation algorithm

Initially the RSS values between the anchor node and unknown node are measured. Secondly the statistical modeling is performed to determine the quality of the channel. The distance between anchor node and unknown node is computed through log normal shadowing path loss model and ITU indoor attenuation model. Finally the accurate distance estimation is done using one-dimension Kalman filter estimator whose iterations are limited by Cramer Rao Bound (CRB) value.

\section{A. Range Measurement and Statistical Modeling}

The Received Signal Strength values between anchor node and unknown node are measured in indoor environment. RSS measured values consist of more error components due to multi-path propagation, fading effects and attenuation. To enhance accuracy in the range measurement, more samples are taken at different time instances for different distances. The commercially available Zigbee series 1 RF module supports 16 numbers of channels in the $2.4 \mathrm{GHz}$ ISM band.
The channels considered for the range measurement are B, C, $\mathrm{D}, \mathrm{E}$ and $\mathrm{F}$ [11]. Statistical modeling is done to evaluate the quality of channel. The various statistical parameters that are evaluated are maximum, minimum, mean, median, standard deviation, lower quantile and upper quantile [12]. The channel that results with low standard deviation compared to other channels is chosen as the best channel.

\section{B. Distance Calculation}

The distance of the unknown node with respect to anchor node can be computed from the ensemble mean RSS value of the best channel. The indoor environment considered is destructive, as it consists of thick and thin walls, wooden and steel furniture, floorings, roofings etc. Further the effect of interference from WLAN and Bluetooth devices present in the environmental set up makes the medium more destructive. So, the effect of path loss, attenuation and interference are also included in calculating the distance parameter [13]. The distance of unknown node is computed using two models namely path loss log normal shadowing and ITU indoor attenuation models. Then the distance error which is the difference between the actual distance and the calculated distance is computed.

1) Log normal shadowing path loss model:

The path loss between node $\mathrm{k}$ and node $\mathrm{u}$ is random and distributed log normally which can be modeled as

$P L\left(d_{k u}{ }^{\prime}\right)[d B]=\overline{P L}\left(d_{r e f}\right)+10 n_{p} \log \left(d_{k u}{ }^{\prime} / d_{r e f}\right)+X_{\sigma}$

where $\overline{P L}\left(d_{r e f}\right)$ is the ensemble path loss at a short reference distance $d_{r e f .} X_{\sigma}$ is the zero mean Gaussian random variable with standard deviation $\sigma$ and $n_{p}$ is path-loss exponent, typically lies between 2 and 4 .

The ensemble mean received power is the difference of the transmitted power and the path loss component at the specific distance $d_{k u}$ ' which is modeled as,

$P_{r}\left(d_{k u^{\prime}}\right)[d B m]=P_{t}(d B m)-P L\left(d_{k u}{ }^{\prime}\right)[d B]$

Substituting eqn (2) in (3),

$P_{r}\left(d_{k u}{ }^{\prime}\right)[d B m]=\left[P_{t}(d B m)-\overline{P L}\left(d_{r e f}\right)-10 n_{p} \log \left(d_{k u}{ }^{\prime} / d_{r e f}\right)-\quad X_{\sigma}\right]$

where $P_{r}\left(d_{k u}{ }^{\prime}\right)$ is the average RSS value in $\mathrm{dBm}$ or received power and $P_{t}(\mathrm{dBm})$ is the power transmitted.

$\overline{P L}\left(d_{r e f}\right)=-10 \log \left(G_{t} G_{r} \lambda^{2} /(4 \pi)^{2} d_{r e f}^{2}\right)$

where $G_{t}, G_{r}=$ Gain of the transmitter and receiver antennas respectively

$\lambda=$ wavelength of the signal by $c / f$

$c=$ velocity of light in $\mathrm{m} / \mathrm{s}$

$f=$ frequency of the signal in $\mathrm{Hz}$.

The distance $d_{k u}$ ' computed from log-normal shadowing model can be expressed as

$$
d_{k u}{ }^{\prime}=d_{r e f} *\left(10^{\left[\left(P t(d B m)-\overline{P L}(d r e f)-X-\operatorname{Pr}\left(d k u^{\prime}\right)[d B m]\right)\right.} \sigma_{\sigma}^{\left(10 n_{p}\right]}\right)
$$

2) ITU Indoor Attenuation Model

The amount of attenuation is predominant in an indoor environment. The range calibration can also be computed by 
an attenuation model. The ITU indoor attenuation model [14] is formally expressed as

$$
\overline{P L}\left(d_{r e f}\right)[d B]=20 \log f+L \log d_{r e f}+P_{f}\left(f_{r k u}\right)-28
$$

where $\overline{P L}\left(d_{\text {ref }}\right)[d B]=$ ensemble mean path loss at the reference distance in $\mathrm{dB}$

$L=$ distance power loss coefficient

$f_{r k u}=$ Number of floors between the node $k$

and node $u$

$P_{f}\left(f_{r k u}\right)=$ floor loss penetration factor.

The distance $d_{k u}$ "' computed from ITU indoor attenuation model can be obtained by substituting eqn. (7) in eqn. (6)

$$
\begin{aligned}
& d_{k u}=\left[d _ { r e f } * \left(10^{[(P t(d B m)-20 \log f-L \log d r e f-P f(f r k u)+}\right.\right. \\
& \left.\left.\left.\left.28-X-\operatorname{Pr}\left(d k u^{\prime}\right)[d B m]\right) /(10 n)\right]\right)\right]
\end{aligned}
$$

\section{Distance Estimation}

The accuracy of the distance calculated by the two models can be improved by minimizing the distance error between the actual distance and the calculated distance. The accurate distance can be estimated through one-dimensional Kalman estimator. The one-dimensional Kalman estimator is modeled as an average filter that combines, the distance values calculated by model 1 and 2 . The distance values for the two models are obtained after statistical modeling by which the best channel selection is done.

Let $d_{k u}$ ' be the range obtained by log-normal shadowing model (Model 1) and $d_{k u}$ " be the range obtained by ITU indoor attenuation model (Model 2). Let $\sigma_{1}$ and $\sigma_{2}$ be the standard deviations obtained from modell and model 2 respectively. Both errors can be combined to get an estimation using Kalman algorithm.

$\sigma_{1}=$ standard deviation for $d_{k u}$ ' about the mean which is given by

$$
\sigma_{1}=\left(\left(\left(d_{k u}{ }^{\prime}-m\right)^{2}+\left(d_{k u}-m\right)^{2}\right) /\left(n_{d}-1\right)\right)^{1 / 2}
$$

where $m=\left(d_{k u}{ }^{\prime}+d_{k u}\right) / 2$

$$
n_{d}=\text { number of samples }
$$

$\sigma_{2}=$ standard deviation for $d_{k u}$ " about the mean which is given by

$$
\sigma_{2}=\left(\left(\left(d_{k u} "-m_{1}\right)^{2}+\left(d_{k u}-m_{1}\right)^{2}\right) /\left(n_{d}-1\right)\right)^{1 / 2}
$$

where $m_{1}=\left(d_{k u}{ }^{\prime \prime}+d_{k u}\right) / 2$

If both models have same standard deviation, then $\sigma_{1}=\sigma_{2}$. If path loss is predominant, then $\sigma_{1}>\sigma_{2}$. Similarly, if attenuation is high, then $\sigma_{1}<\sigma_{2}$. The complexity of the proposed algorithm increases if the error in the calculated distance increases. The iterations can be limited by the amount of errors. If $\sigma_{1=} \sigma_{2}$, the estimated distance will be the average of $d_{k u}$ ' and $d_{k u}$ ". If $\sigma_{1}>>\sigma_{2}$, then $\mathrm{d}_{\mathrm{ku}}$ " can be taken as the estimated distance. If $\sigma_{1}<<\sigma_{2}$, then $\mathrm{d}_{\mathrm{ku}}$ ' can be taken as the estimated distance [14].

In any other case, the weighted average of the distances calculated from both the models are used to estimate $d_{k u}$, which may be called as $\hat{d}$

$$
\hat{d}_{k u}=\left(\frac{d_{k u}{ }^{\prime}}{\sigma_{1}^{2}}+\frac{d_{k u}{ }^{\prime}}{\sigma_{2}{ }^{2}}\right) /\left(\frac{1}{\sigma_{1}^{2}}+\frac{1}{\sigma_{2}^{2}}\right)
$$

or

$$
\hat{d}_{k u}=\left(d_{k u}{ }^{\prime} \sigma_{2}^{2}+d_{k u}{ }^{\prime \prime} \sigma_{1}^{2}\right) /\left(\sigma_{1}^{2}+\sigma_{2}^{2}\right)
$$

The above estimate can also be rewritten as

$$
\hat{d}_{k u}=d_{k u}{ }^{\prime}+K\left(d_{k u},{ }^{\prime}-d_{k u}{ }^{\prime}\right)
$$

where $K=\sigma_{1}^{2} /\left(\sigma_{1}^{2}+\sigma_{2}^{2}\right)$ is defined as the Kalman gain.

The accuracy of the estimator $\hat{d}_{k u}$ can be determined based on Cramer Rao bound value [3, 5].

The CRB value is given below,

$$
\text { CRB bound }=\frac{\sigma^{2}}{n_{d}}
$$

where $\sigma=\left(\sigma_{1}+\sigma_{2}\right) / 2$

The iterations of the estimator is repeated till following condition is satisfied,

$$
\operatorname{Var}\left(\hat{d}_{k u}\right) \geq C R B \text { bound }
$$

The estimated distance for every iteration is compared with the actual distance. The iterations are repeated till the variance reaches the CRB bound. The value obtained in each iteration of the proposed algorithm is given in TABLE I.

TABLE I. ITERATION ALGORITHM OF KALMAN FILTER

\begin{tabular}{lllll}
\hline \hline \multirow{2}{*}{ Actual Distance } & CRB & Iteration 1 & $\begin{array}{l}\text { Iterat } \\
\text { ion 2 }\end{array}$ & $\begin{array}{l}\text { Final } \\
\text { distance }(\mathrm{m})\end{array}$ \\
\hline
\end{tabular}

$\begin{array}{lllll}2 & 0.0553 & 0.0603 & 0.05 & 2.0502 \\ 4 & 0.2607 & 0.1524 & - & 3.8476 \\ 6 & 0.7601 & 0.5529 & - & 5.4471 \\ 8 & 1.0751 & 0.3507 & - & 7.6493 \\ 10 & 1.3050 & 0.0298 & - & 10.029\end{array}$

\section{Simulation Results}

The experimentation is done in indoor environment using zigbee series $1 \mathrm{RF}$ module and the associated X-CTU software of MAXSTREAM. 20 samples of RSS (Received Signal Strength) values are measured at 20 different time instances for a specific distance. The experiment has been repeated for five different channels (B, C, D, E and F) with five different frequencies. 
Fig. 2 illustrate the relationship between distance and RSS measurement for channels B. It is seen that the Received Signal Strength of the unknown node decreases as distance between the anchor and unknown node increases. Similar relationship can be obtained for the remaining channels.

Fig. 3 shows the statistical modeling of channel B in which the various statistical parameters like minimum, maximum, mean, standard deviation, lower quantile and upper quantile are computed with the measured RSS values.

RSS vs Distance for channel B

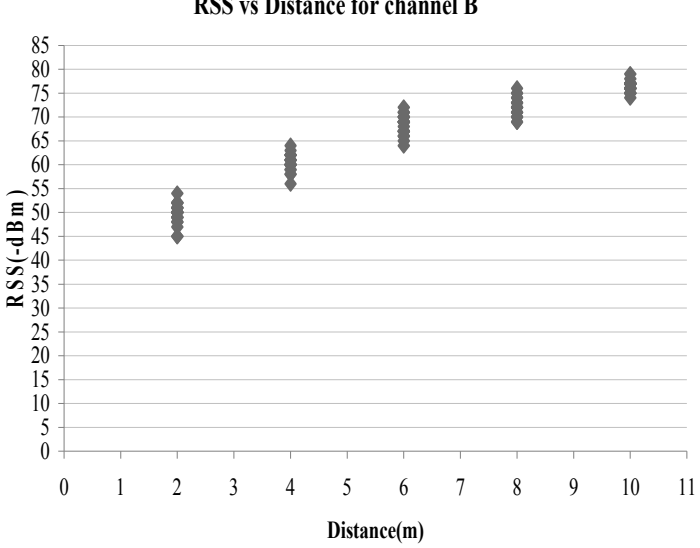

Fig. 2. RSS vs Distance in Channel B

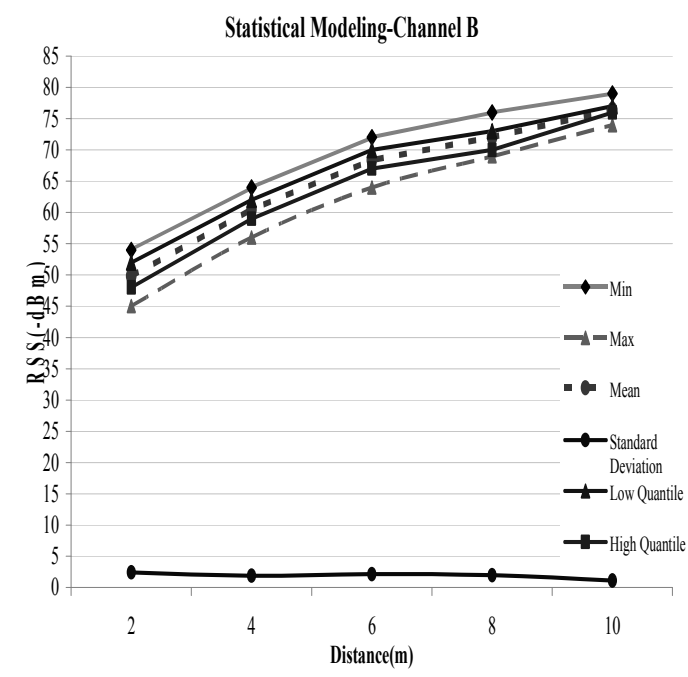

Fig. 3. Statistical modeling of channel B

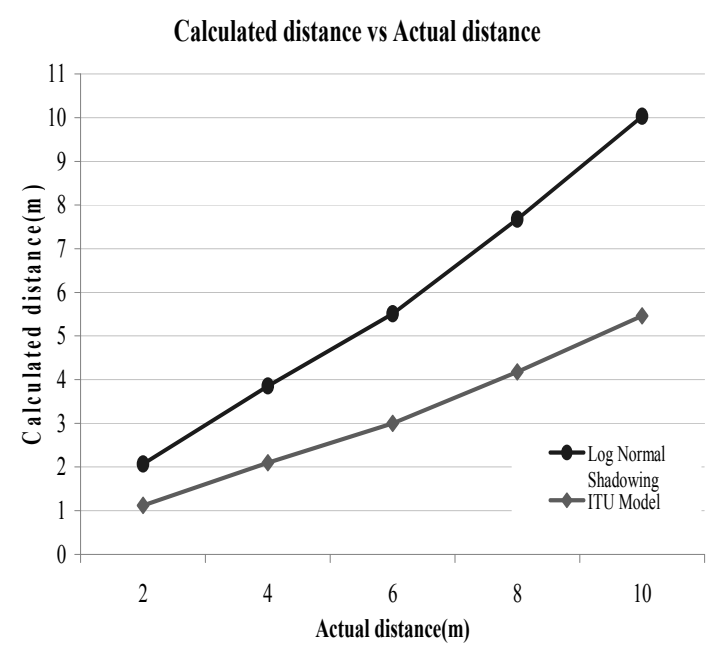

Fig. 4. Calculated distance vs actual distance

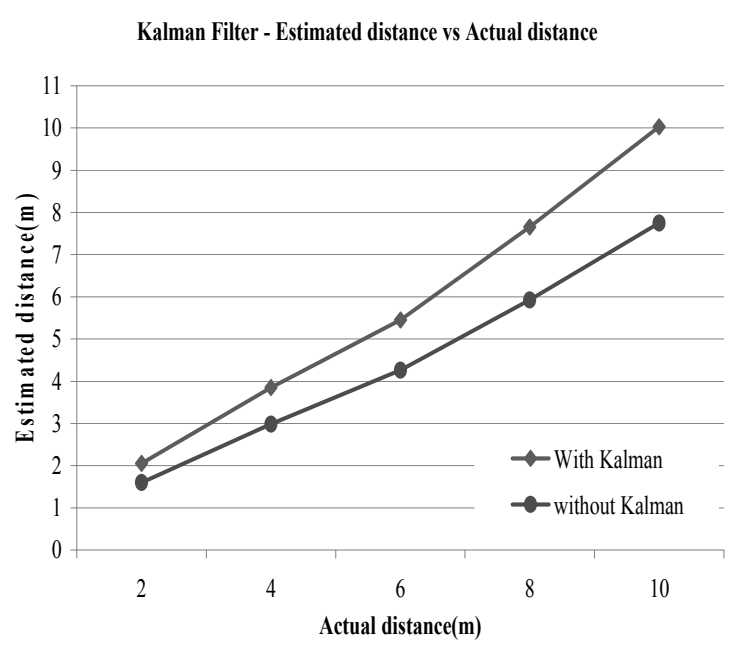

Fig. 5. Comparison of distance estimation

TABLE II. STANDARD DEVIATION VALUES OF RSS MEASUREMENTS FOR DIFFERENT CHANNELS

\begin{tabular}{|c|c|c|c|c|c|}
\hline $\begin{array}{l}\text { Dist } \\
\text { (m) }\end{array}$ & $\begin{array}{l}\text { B } \\
(2.404-2 . \\
406) \\
\text { GHZ }\end{array}$ & $\begin{array}{l}\mathrm{C} \\
(2.409-2 . \\
411) \\
\mathrm{GHz}\end{array}$ & $\begin{array}{l}\text { D } \\
(2.414-2 . \\
416) \\
\mathrm{GHz}\end{array}$ & $\begin{array}{l}\mathrm{E} \\
(2.419-2 \\
.421) \\
\mathrm{GHz}\end{array}$ & $\begin{array}{l}\mathrm{F} \\
(2.424- \\
2.426) \\
\mathrm{GHz}\end{array}$ \\
\hline 2 & 2.39 & 2.13 & 2.62 & 0.92 & 1.69 \\
\hline 4 & 1.87 & 1.65 & 1.53 & 1.23 & 1.38 \\
\hline 6 & 2.13 & 1.67 & 1.26 & 1.37 & 2.05 \\
\hline 8 & 1.98 & 1.29 & 1.04 & 1.31 & 1.92 \\
\hline 10 & 1.11 & 1.44 & 0.96 & 0.61 & 1.18 \\
\hline
\end{tabular}

TABLE III. ONE-DIMENSION KALMAN ESTIMATOR

\begin{tabular}{|c|l|l|l|l|l|}
\hline $\begin{array}{l}\text { Act } \\
\text { dist. } \\
(\mathrm{m})\end{array}$ & $\begin{array}{l}\text { Err } \\
\text { (without } \\
\text { KF) }\end{array}$ & $\begin{array}{l}\text { \% of } \\
\text { error } \\
\text { with-out } \\
\text { KF }\end{array}$ & $\begin{array}{l}\text { Err } \\
\text { KF) } \\
\text { KF }\end{array}$ & $\begin{array}{l}\text { \% of } \\
\text { error } \\
\text { with KF }\end{array}$ & $\begin{array}{l}\text { of } \\
\text { imp. } \\
\text { Acc. }\end{array}$ \\
\hline 2 & 0.4705 & 23.5 & 0.0502 & 2.51 & 89 \\
\hline 4 & 1.0213 & 25.53 & 0.1524 & 3.81 & 85 \\
\hline 6 & 1.7437 & 29.06 & 0.5529 & 9.21 & 68 \\
\hline 8 & 2.0737 & 25.92 & 0.3507 & 4.38 & 83 \\
\hline 10 & 2.2848 & 22.85 & 0.0298 & 0.29 & 98 \\
\hline
\end{tabular}

TABLE IV. COMPUTATIONAL COMPLEXITY

\begin{tabular}{|c|c|c|c|}
\hline \multirow{2}{*}{$\begin{array}{c}\text { Equation } \\
\text { No. }\end{array}$} & \multicolumn{3}{|c|}{ Operations } \\
\cline { 2 - 4 } & Divisions & Additions & Loop \\
\hline 6 & 1 & 3 & --- \\
\hline 8 & 1 & 6 & --- \\
\hline 11 & 5 & 2 & I* \\
\hline
\end{tabular}


Similar analysis can be performed for the other channels as shown in Table 2.

Here the standard deviation is considered as the deciding factor to quantify the quality of the channel. It is found that the channel E can be selected as the best channel as it possesses low standard deviation. The distance of the unknown node is calculated by both log normal shadowing path loss model with path loss exponent $n_{p}=4$ and ITU indoor attenuation model.

Fig. 4 illustrates the calculated distance obtained by both the models. The distance error is computed by both the models. It is found that the distance error computed by $\log$ normal shadowing model is less compared to ITU indoor attenuation model. It is due to the value chosen for path loss exponent that reflects the nature of the multipath propagation medium. Figure 5 shows distance estimation with and with out one-dimensional Kalman filter. Table 3 presents the results of Kalman estimated distance and the associated error percentage. It can be seen that the error has been drastically reduced using Kalman estimator.

The computational complexity of the proposed algorithm is estimated as given in Table 4. So, the total computation is $(9 \mathrm{~A}+2 \mathrm{D})+(2 \mathrm{~A}+5 \mathrm{D})$ I. The division operations involved in equation 6,8 and 11 can be converted into multiplications by performing the division once and saving the quotient in temporary memory location to reduce the computational complexity. As division takes more time than addition and multiplication, computation of the proposed algorithm will be $\mathrm{O}((2 \mathrm{~A}+\mathrm{I}(5 \mathrm{D}))$ where $\mathrm{I}$ is the number of iteration which is limited by the CRB value.

The computational complexities involved in related works are less when compared to the proposed algorithm. However the accuracy of distance value is improved in the proposed algorithm when compared to the existing schemes through one-dimensional Kalman estimator. The complexity of the existing schemes is increased in the coordinate estimation phase as the amount of distance errors is more which is reduced in the proposed algorithm. In the proposed algorithm, no special hardware is used to measure RSS value, which results in reduction of hardware complexity compared to that of [4].

The time complexity involved in the proposed scheme is also reduced as only three anchor nodes are needed at the maximum to estimate the location of the unknown node [13].

\section{CONCLUSION}

In this paper, a novel cost-effective accurate distance estimation algorithm is proposed. It uses path loss and attenuation models to compute the distance of the nodes accurately. The error is minimized with one-dimensional Kalman algorithm in which two models are integrated to get better results. Further the one-dimensional Kalman is not computationally heavy. The algorithm will be extended in future to determine the coordinates of the unknown nodes by employing lateration techniques.

\section{ACKNOWLEDGEMENT}

The authors would like to thank Tata Consultancy Services (TCS) for funding this project.

\section{REFERENCES}

[1] J. Yick, B. Mukherjee and D. Ghosal (2008), "Wireless sensor network survey," ELSEVIER Journal on Computer Networks, Vol. 52, pp. 2292-2330.

[2] H. Karl and A. Willig (2006), "Protocols and Architectures for Wireless Sensor Networks," Wiley Publications.

[3] N. Patwari and A. O. Hero (2006), "Demonstrating Distributed Signal Strength Location Estimation," SenSys'06, pp.353-354.

[4] R. Rojas (2003), "The Kalman Filter," Technical report, Freie University of Berlin, 2003.

[5] E. G. Larsson (2004), "Cramer-Rao bound analysis of distributed positioning in sensor networks," IEEE Signal Processing Letters, Vol. 11,No. 3,pp.334-337.

[6] Timmermann.D, C. Buschmann and J. Koberstein (2006), "Minimal Transmission Power as Distance Estimation for Precise Localization in Sensor Networks," IWCMC'06, pp.1331-1336.

[7] P. K Sahoo, I. S.Hwang and S. Y Lin (2008), "A Distributed Localization Scheme for Wireless Sensor Networks," Internation Conference On Mobile Technology, Applications, And Systems, Vol:6, Article No. 77, No: 7, pp.1031-1050.

[8] D. Zhao, Y. Men and L. Zhao (2007), “A hop-distance algorithm for self-localization of wireless sensor networks," International conference on software engineering, artificial intelligence and parallel/distributed computing, Vol.2, IEEE computer society, pp.108-112.

[9] M. Sugano, T. Kawazoe, Y. Ohta, and M. Murata (2006), "Indoor localization system using RSSI measurement of wireless sensor network based on ZIGBEE standard," International Conference on Wireless Sensor Networks, pp.6-12.

[10] S. Qin-Qin, H. Hong, F. Tao and L. De-Ren (2006), "Using Linear Intersection for Node Location Computation in Wireless Sensor Networks," Acta Automatica Sinica, Vol. 32, No. 6, pp.907-914.

[11] www.digi.com, "Support/k-base/XCTU software," Aug 5, 2009.

[12] A. Awad, T. Frunzke, and F. Dressler (2007), "Adaptive distance estimation and localization in WSN using RSSI measures," Proceedings of 10th EUROMICRO Conference on Digital System Design - Architectures, Methods and Tools, pp. 471-478.

[13] Theodore S. Rappaport, "Wireless Communications-Principles and Practice," Edition II, Prentice-Hall of India Publications.

P. T. V. Bhuvaneswari completed her B.E (EEE) from St. Peters Engineering College, Madras University, Chennai and M.E (Applied Electronis) from Government College of Engineering, Bharathiyar University, Coimbatore and Ph.D in the area of Wireless Sensor Network from Madras Institute of Technology, Anna University, Chennai. She has successfully completed funded project sponsored by Tata Consultancy Services. She is currently doing a project for NRDMS,DST, consultancy of india. She has guided several Under-graduates and Post-graduates. She has several publications in conferences and journals. She is currently working as Assistant Professor in Electronics Department and her areas of interest are wireless sensor network, wireless networks, wireless and mobile communications.

V. Vaidehi completed her B.E (ECE) from college of Engineering, Guindy, M.E and Ph.D from Madras Institute of Technology, Anna University, Chennai, INDIA. She has successfully completed several funded projects and guided several M.S/M.E and Ph.Ds. She has several publications in conferences and journals. She has served as a member of Board of Studies, Head computer centre, Information Technology and Electronics Department. She is currently working as professor in Electronics Department, Madras Institute of Technology and her areas of interest are Wireless Networks, Advanced Computer Architecture and Real-time Embedded Systems. 